\title{
First passage and excursion time models for valuing defaultable bonds
}

\author{
Martina Nardon \\ Department of Applied Mathematics \\ University Ca' Foscari of Venice \\ Dorsoduro, $3825 / \mathrm{E}$ \\ 30123 Venice - Italy \\ mnardon@unive.it
}

October 28, 2005

\begin{abstract}
In this contribution, we study structural models of defaultable bond pricing in which default occurs at the first time a relevant process either reaches the default boundary or has spent continuously (or cumulatively) a fixed time period below that threshold. Unlike first-passage time approaches, excursion time models allow for a non-absorbing state of default. Both the first-passage time and the excursion time approaches can be generalized by defining the default time as the first instant at which the firm value process (or another signaling process) either remains a certain time below the default threshold or hits a lower barrier. This corresponds, for instance, to a situation in which a firm is allowed temporarily to be short of funds, but enters default immediately when the financial distress becomes severe. Moreover, we examine the effects of different default time specifications on bond prices and credit spreads.
\end{abstract}

Keywords. Credit risk, structural models, default boundary, firstpassage time, excursion time.

(J.E.L. Classification: C15, C63, G12, G13.)

\section{Introduction}

Assessing default risk and the timing of such an event is crucial in order to evaluate the prices and returns of bonds. Although the effects of credit risk on bond values have long been analyzed, only relatively recently analytical models were developed in order to study and quantify these effects. 
A rational theory of credit risk is based on the pathbreaking model of Merton (1974). Since then, two main streams of thought have been developed: structural models, based on a balance-sheet notion of solvency and option pricing theory, and reduced-form (or intensity-based) models of credit risk.

In this contribution, we study structural models of defaultable bond pricing in which the debtor's inability (or willingness) to pay is explicitly modeled. A firm defaults when it cannot meet its financial obligations. Structural approach is based in an explicit manner on the timing of such an event: default happens when assets are too low relative to liabilities, depending on some critical level being attained.

Çetin et al. (2004) argue that managers of a firm should know if default is about to occur. From managers' perspective, default is a predictable event. This perspective is also consistent with the formulation of structural credit risk models, in which the time of default is an accessible stopping time.

On the other hand, the market has partial information ${ }^{1}$, which makes default a surprise and the default time not predictable ${ }^{2}$. This latter perspective is consistent with reduced form credit risk models.

In the Black-Scholes-Merton model default may occur at the maturity date in the event that the issuer's assets are less than the face value of the debt. While first-passage models are based on the assumption that default occurs as soon as the asset value of the firm falls below a certain threshold. Such a boundary is often set equal to the face value of the firm's liabilities. Black and Cox (1976) introduce the idea that default can occur at the first time that assets drop to a sufficiently low level, either before or at the maturity of the debt. They assume a simple time-dependent default boundary.

First-passage time models have been widely studied and developed in the literature. A list of important contributions in this field includes (but it is not limited to) Kim et al. (1993), Shimko et al. (1993), Longstaff and Schwartz (1995), Bryis and de Varenne (1997), Cathcart and El-Jahel (1998), Taurén (1999), Collin-Dufresne and Goldstein (2001), Hsu et al. (2003), and Hui et al. (2003).

First-passage approach makes no distinction between the time at which a firm enters financial distress and the time at which it is either liquidated or a reorganization plan is accepted and the firm emerges from bankruptcy. In the classical approach, a firm defaults when its assets are too low according to some measure. Consequently the firm stops operating and is immediately liquidated. This definition of default seems not reflecting economic reality: bankruptcy laws often grant an extended time period for restructuring (see Eberhart et al. (1999) and Helwege (1999)).

\footnotetext{
${ }^{1}$ Or it has the managers' information set plus a noise (see Duffie and Lando (2001)), or leaves any knowledge of firm's value apart, by considering default as an exogenous event.

${ }^{2}$ The time of default becomes inaccessible, which means that one is not able to anticipate default.
} 
Recently there has been some interest in the credit risk literature in models in which the duration of the default (the time the firm spends in bankruptcy before being reorganized or liquidated) is explicitly modeled. Such models involve stopping times related to excursions and hence we will refer to as excursion time models. In the excursion approach the default time is defined as the first instant at which the process that governs default has spent continuously (or cumulatively) a fixed time period (called the window) below the default boundary. These models are based on the theory of valuation of Parisian options (see, e.g., Chesney et al. (1997), and Haber et al. (1999)) and occupation time derivatives (Hugonnier (1999)). Unlike first-passage time approach, the excursion time approach allows for a non-absorbing state of default. While Moraux (2003) points out that only occupation time (cumulative excursion time) models describe the whole past financial distress. Such an approach considers the total time spent by the value process beyond the default threshold over the monitored time period, hence it seems more suitable in order to study the financial history and distress periods of the debt issuer.

Both the first-passage time and the Parisian time approaches can be generalized by defining the default time as the first instant at which the firm value process (or another signaling process) either remains a certain time below the default threshold or hits a lower barrier. This corresponds, for instance, to a real situation in which a firm is allowed temporarily to be short of funds, but enters default immediately when the financial distress becomes severe and a critical level, defined as a threshold set below the classical default boundary, is reached.

In this contribution, we provide a review of first-passage and excursion time models for credit risk. We then propose a generalization of both the first-passage and excursion approaches, which takes into consideration the severity of the financial distress. The main focus is on the definition of the timing of default. Moreover, we examine the effects of different default time specifications on bond prices and credit spreads, under the assumptions of a particular structural model. To this aim, in the absence of closed form solutions, in general, numerical procedures are required, in particular when some simplifying assumptions regarding default-free interest rate modeling are relaxed. Monte Carlo simulation can be used in order to estimate the default probability.

An outline for this paper is as follows. Section 2 reviews first-passage time models and recalls some well known results. Excursion time approach is analyzed in Section 3. In Section 4, a new formulation of the timing of default is introduced, which extends both the first-passage and excursion time definitions. Section 5 presents some results of the simulation analysis. Section 6 concludes. 


\section{$2 \quad$ First-passage time models}

In the Black-Scholes-Merton model default may occur only at the maturity of the debt. In first-passage time models, introduced by Black and Cox (1976), this assumption is relaxed to allow for early default. More precisely, the time of default is defined as the first instant at which a relevant process, describing for instance the assets value of the firm, falls below a certain level called the default boundary (or default barrier). Such a boundary can be assumed as a constant threshold (as, e.g., in the contribution by Longstaff and Schwartz (1995)), a time-dependent barrier (Black and Cox (1976) consider an exponential default boundary), or it can be governed by some stochastic process (see, e.g., Saá-Requejo and Santa-Clara (1999)) ${ }^{3}$. A possible economic interpretation of the default boundary is the presence of some safety covenants in the contract (as it is the case in Black and Cox (1976)). This boundary may be given exogenously or determined endogenously, as the solution of an optimal decision problem ${ }^{4}$.

When the firm value falls below the distress threshold, the firm enters bankruptcy and may be either reorganized or liquidated. This point is crucial: in first-passage time modeling of credit risk no distinction is made between the time at which a firm enters bankruptcy (under Chapter 11) and the time at which it is either liquidated or the reorganization plan is accepted and the firm exits bankruptcy. In the excursion time approach, which will be discussed in Section 3, the duration of the default (the time the firm spends in bankruptcy) is expressly modeled.

In this section, we take into consideration first-passage time models. Formally, let us consider a continuous time model on the time horizon $[0, \bar{T}]$. Assume that a probability space $(\Omega, \mathcal{F}, \mathbb{P})$ is equipped with a filtration $\left(\mathcal{F}_{t} ; t \geq 0\right)$, provided some technical conditions, where $\mathcal{F}_{t}$ represents the information set available at time $t$.

In a contingent claims framework (with full information), let us consider a firm with a single issue of debt in the form of a zero-coupon bond (henceforth $\mathrm{ZCB}$ ) promising to pay the face value (which is assumed to be one euro) at the maturity date $T$. Let $v(t, T)$ denote the price at time $t \leq T$ of a defaultable ZCB with maturity $T$. Assume, moreover, that default-free zerocoupon bonds are traded for all maturities, and let $r$ denote the default-free spot interest rate ${ }^{5}$. Both defaultable and default-free bonds markets are assumed to be arbitrage-free, so there exists an equivalent martingale measure $\mathbb{Q}^{6}$.

\footnotetext{
${ }^{3}$ Note that in Merton's model the default boundary is defined only at maturity, hence it reduces to a single point.

${ }^{4}$ See, among others, Leland (1994), and Leland and Toft (1996).

${ }^{5}$ For the moment, no assumption is made about the spot rate dynamics. $r$ will be either a constant parameter, a time-varying function, or governed by some stochastic process.

${ }^{6}$ This probability measure may not be unique (see Jarrow and Protter (2004)).
} 
In this setting, consider a stochastic process $X=\left(X_{t}\right)_{t \geq 0}$ (continuous and adapted), which describes the firm's value (or another relevant firm's feature, as assets value, leverage ratio, operating cash flows, etc.), and the default boundary $H=\left(H_{t}\right)_{t \in[0, T]}$. Then, the information set at time $t$ will be $\mathcal{F}_{t}=\sigma\left(X_{u} ; u \leq t\right)$. Note that, when the default boundary is modeled as a stochastic process, the information set is $\mathcal{F}_{t}=\sigma\left(X_{u}, H_{u} ; u \leq t\right)$.

As regards the recovery value upon default, different formulations are possible. In practice, when a firm enters default and is then liquidated, bondholders receive a variety of securities (different combinations of cash, zero-coupon or coupon bearing bonds, shares, warrants, and convertible securities). Here we make some simplifying assumptions (in line with Jarrow and Protter (2004)): bondholders may receive, for instance, the value of the boundary, which could be paid either at the default time or at maturity $T$. As an alternative, creditors may receive a fraction (which may be a parameter specified exogenously or even a random variable) of an equivalent default-free ZCB maturing at time $T^{7}$.

Before going further, we recall some theoretical notions on random times (see Protter (2004)).

Definition 1 Given a complete probability space $\{(\Omega, \mathcal{F}, \mathbb{P})\}$, equipped with a filtration $\left(\mathcal{F}_{t}\right)_{t \geq 0}$, a random variable $\tau: \Omega \rightarrow[0, \infty]$ is a stopping time if the event $\{\omega \in \Omega: \tau(\omega) \leq t\} \in \mathcal{F}_{t}$, for every $t \geq 0$.

Definition 2 A stopping time $\tau$ is predictable if there exists a sequence of stopping times $\left(\tau_{n}\right)_{n \geq 1}$ such that $\tau_{n}$ is increasing, $\tau_{n}<\tau$ on $\{\tau>0\}$, for all $n$, and $\lim _{n \rightarrow \infty} \tau_{n}=\tau$ a.s.

The sequence $\left(\tau_{n}\right)$ is said to announce $\tau$.

In first-passage time models, the time of default is a random variable whose distribution is that of the first-hitting or first-passage time of the relevant process $X$ through the default boundary $\left(H_{t}\right)_{t \in[0, T]}$. Formally,

$$
\tau^{H}=\inf \left\{t \in[0, T): X_{t}=H_{t}\right\}=\inf \left\{t \in[0, T): X_{t} \leq H_{t}\right\},
$$

with $\inf \emptyset=\infty$.

It turns out that, the random time $\tau^{H}$ is a predictable stopping time ${ }^{8}$, which means that the time of default is not completely a surprise in firstpassage time models, but can be anticipated in some sense by observing the trajectory taken by the process $X$. This feature will be exploited in the simulation analysis in order to estimate the average default time and the probability of default.

\footnotetext{
${ }^{7}$ Guha and Sbuelz (2003) study different recovery forms upon default. See also Bielecki and Rutkowski (2002) and Schmid (2004).

${ }^{8}$ Jarrow and Protter (2004) point out that this property no longer holds, in general, when the process $X$ presents jumps.
} 
Of course, at maturity $T$, the default boundary has to be specified in a proper way to avoid inconsistencies (see Giesecke (2004)). At maturity, in general default occurs if assets value is less than the face value of the debt, denoted by $F$ (hence $H_{T}=F$ ). Let $\tau_{T}$ denote the random variable defined

$$
\tau_{T}= \begin{cases}T & \text { if } X_{T} \leq F \\ \infty & \text { otherwise }\end{cases}
$$

The default time, denoted by $\tau^{*}$, is defined as the minimum between the first hitting time $\tau^{H}$ and $\tau_{T}$,

$$
\tau^{*}=\tau^{H} \wedge \tau_{T} .
$$

As an example, if we assume that the interest rate $r$ is constant, and the recovery value upon default is equal to the barrier value at default, then the price at time $t$ of a defaultable ZCB with maturity date $T$ is given by

$$
v(t, T)=\mathbb{E}_{t}^{\mathbb{Q}}\left(e^{-r(T-t)}\left(\mathbf{1}_{\left\{\tau^{H} \leq T\right\}} H_{\tau^{H}}+\mathbf{1}_{\left\{\tau^{H}>T\right\}} 1\right)\right),
$$

where $\mathbb{E}_{t}^{\mathbb{Q}}(\cdot)$ denotes expectation under the probability measure $\mathbb{Q}^{9}$, conditional to the information at time $t$ (and conditional on no default until time $t$ ), and $\mathbf{1}_{A}$ is the indicator function of $A$.

$v(t, T)$ in equation (4) can be evaluated analytically in some special cases. For instance, let $X$ satisfy the following stochastic differential equation

$$
\frac{d X_{t}}{X_{t}}=r d t+\sigma d W_{t}, \quad X_{0}>0
$$

where $r>0, \sigma>0$, and $W=\left(W_{t}\right)_{t \geq 0}$ is a standard Wiener process under the risk neutral measure. If we assume that the default threshold is constant, $H_{t}=H$ for every $t \in[0, T]$, then we have the following well known result (see Bielecki and Rutkowski $(2002))^{10}$ for the default probability $\mathbb{Q}_{t}\left(\tau^{H} \leq T\right)$ and the defaultable $\mathrm{ZCB}$ value

$$
v(t, T)=H e^{-r(T-t)} \mathbb{Q}_{t}\left(\tau^{H} \leq T\right)+e^{-r(T-t)}\left[1-\mathbb{Q}_{t}\left(\tau^{H} \leq T\right)\right],
$$

where

$$
\begin{aligned}
\mathbb{Q}_{t}\left(\tau^{H} \leq T\right)=\mathcal{N}\left(h_{1}\right)+\left(\frac{X_{t}}{H}\right)^{-2 \frac{\left(r-\sigma^{2} / 2\right)}{\sigma^{2}}} \mathcal{N}\left(h_{2}\right), \\
h_{1}=\frac{-\log \left(X_{t} / H\right)-\left(r-\sigma^{2} / 2\right)(T-t)}{\sigma \sqrt{T-t}}, \\
h_{2}=\frac{-\log \left(X_{t} / H\right)+\left(r-\sigma^{2} / 2\right)(T-t)}{\sigma \sqrt{T-t}},
\end{aligned}
$$

\footnotetext{
${ }^{9}$ Sometimes we will write simply $\mathbb{E}_{t}(\cdot)$.

${ }^{10}$ See also Giesecke (2004), and Moraux (2004).
} 
and $\mathcal{N}(\cdot)$ is the standard normal distribution function.

In the absence of closed form solutions, one must resort to numerical approximations and computational techniques, in particular when some simplifying assumptions regarding default-free interest rate dynamics are relaxed. Monte Carlo simulation can be used in order to estimate the default probability (see Hsu et al. (2003)).

In the rest of this section, some well known first-passage time models proposed in the literature are briefly reviewed. Distinguishing models' features are based on the assumptions made about the following crucial elements: the modeling of the process that governs default, the (possibly random) variable that describes the loss given default (or, alternatively, the recovery value upon default), and the dynamics of the default-free interest rate.

\subsection{A model with time-varying default boundary and constant default-free interest rate}

Black and Cox (1976) extend Merton's model to allow for early default. In this section we summarize in brief the main features of their model.

Let us consider a perfect, frictionless market in which trading takes place at continuous time. Let $r$ be the risk-free interest rate, which is assumed to be constant. Consider a firm with outstanding only equity, and that issues a single bond with a promised payment $F$ at maturity $T$.

Denote with $V=\left(V_{t}\right)_{t \geq 0}$ the stochastic process that drives the value of the firm. Under the risk neutral measure, $V$ satisfies the following stochastic differential equation

$$
d V_{t}=(r-\delta) V_{t} d t+\sigma V_{t} d W_{t},
$$

where $\delta$ is the constant payout rate, $\sigma>0$, and $W$ is a standard Wiener process.

Until the maturity of the debt, $V$ may take on high values, or it may fall down to a critical level at which the debtor is no longer able to meet its financial obligations. There may be some upper and lower barriers (given exogenously or endogenously determined) in correspondence to which the firm must take on specific values. Here we consider only the case of a lower boundary which can be interpreted as some safety covenant. Safety covenants (see Black and Cox (1976)) are contractual provisions which give the bondholders the right to force firm into bankruptcy when the firm's value is insufficient to repay the debt. In this model, the timing of default is defined as the first instant at which the safety covenant is violated by the process $V$,

$$
\tau^{H}=\inf \left\{t>0: V_{t} \leq H_{t}\right\} .
$$

An important element in first-passage time models is the specification of the default boundary. Black and Cox (1976) adopt a time-varying default 
barrier; more precisely, the default boundary value at time $t$ is

$$
H_{t}=H e^{-\gamma(T-t)},
$$

where $H$ and $\gamma$ are parameters, with $H>0$.

The recovery value in the event of premature default is assumed to be equal to the bankruptcy level at default, $V_{\tau^{H}}=H_{\tau^{H}}$.

Let $v(V, t, T)$ be the value of the corporate bond at time $t, v$ depends on the value of $V$ and satisfies the following partial differential equation

$$
\frac{1}{2} \sigma^{2} V^{2} \frac{\partial^{2} v}{\partial V^{2}}+(r-\delta) V \frac{\partial v}{\partial V}-r v+\frac{\partial v}{\partial t}=0,
$$

with boundary conditions

$$
\begin{aligned}
& v(V, T, T)=\min (V, F), \\
& v(H, t, T)=H e^{-\gamma(T-t)} .
\end{aligned}
$$

Similar conditions can be expressed for the value of the stock.

In Black and Cox model, the probability of default $\mathbb{Q}_{t}\left(\tau^{H} \leq T\right)$ is defined as the probability of the event $\left\{V_{t} \leq H_{t}\right\}$, for $t \in[0, T]$ (conditional on the fact that the boundary has not been reached yet), where the default boundary is specified as an exponential function of time. This probability can be evaluated analytically:

$$
\begin{aligned}
\mathbb{Q}_{t}\left(\tau^{H}\right. & \leq T)=1-\mathcal{N}\left(\frac{\ln \left(V_{t} / H_{t}\right)+\left(r-\delta-\frac{1}{2} \sigma^{2}\right) \tau}{\sigma \sqrt{\tau}}\right)+ \\
& +\left(\frac{V_{t}}{H_{t}}\right)^{1-2(r-\delta-\gamma) / \sigma^{2}} \mathcal{N}\left(\frac{-\ln \left(V_{t} / H_{t}\right)+\left(r-\delta-\frac{1}{2} \sigma^{2}\right) \tau}{\sigma \sqrt{\tau}}\right)
\end{aligned}
$$

where $\tau=T-t$, and $H_{t}=H e^{-\gamma(T-t)}$.

Black and Cox obtain a formula for the value of the defaultable bond $v(V, t, T)$. In particular, it turns out that $v$ is an increasing function of $V$ and $t$, while it is decreasing in $r, \sigma$ and $\delta$ (see Black and Cox (1976) for details).

An interesting specification of the boundary is

$$
H_{t}=\rho F e^{-r(T-t)},
$$

with $0 \leq \rho \leq 1$. The default threshold at time $t$ is a constant fraction of the face value of the debt $F$, discounted at the risk-free rate $r$. As a result, $v$ is an increasing and convex function of the parameter $\rho$. 


\subsection{A model with constant default boundary and stochastic default-free interest rate}

In this section we consider a first-passage time approach in which the default boundary is assumed to be constant and the hypothesis of deterministic default-free interest rate is relaxed. We shortly present the framework of Longstaff and Schwartz (1995) to the valuation of defaultable bonds, and we describe more in detail the model proposed by Cathcart and El-Jahel (1998).

As Longstaff and Schwartz (1995) observe, the assumption of constant interest rates is difficult to justify in a model to valuing risky corporate bonds that incorporates both credit risk and interest rate risk. In their model, the process $V$ specifies the value of the firm's assets, whose dynamics is driven by a geometric Brownian motion. Let $r=\left(r_{t}\right)_{t \geq 0}$ denote the default-free short-term interest rate, $r$ is assumed to follow a Ornstein-Uhlenbeck process (according to Vasicek (1977)). In a risk-neutral framework, the drift of the process $V$ should depend on $r$.

Furthermore, Longstaff and Schwartz assume there is a constant barrier $H$, which is exogenously assigned, at which firm enters financial distress. If $V$ hits the critical level $H$, the firm immediately defaults (on all debt contracts simultaneously) and it is either liquidated or restructured.

A crucial assumption made by Longstaff and Schwartz (1995) concerns the recovery value upon default. Debt holders receive $1-w$ default-free ZCBs with the same maturity and face value of the corporate debt. The percentage of the writedown $w$ is assumed constant.

In particular, this assumption allows to valuing securities (even complex liabilities) independently from other liabilities issued by the firm. Coupon bearing bonds can be priced in an easy way, as a portfolio of ZCBs.

The model proposed by Cathcart and El-Jahel (1998) is in line with that of Longstaff and Schwartz (1995). The authors introduce the idea of a signaling process whose dynamics governs default. More precisely, default occurs at the first instant the signaling process hits a constant default threshold.

Consider a perfect frictionless market. Let $X=\left(X_{t}\right)_{t \geq 0}$ denote the riskneutralized process that signals default, and assume it satisfies the stochastic differential equation

$$
d X_{t}=\alpha X_{t} d t+\sigma_{X} X_{t} d W_{t}^{X} \quad X_{0}>0,
$$

where $\alpha$ and $\sigma_{X}>0$ are constant parameters, and $W^{X}$ is a standard Wiener process.

Note that, in this setting, the assumption of a constant drift, not depending on $r$, is justified by the fact that the signaling process is not necessarily a value process. The signaling process should be able to describe a variety of effects that can determine default, like for example shortage of funds. 
The authors point out that this formulation is also suitable for sovereign institutions, for which it would be difficult to identify underlying assets.

The default-free spot rate $r$ is assumed to follow a mean reverting square root process (according to Cox, Ingersoll and Ross (1985) model, henceforth CIR). Its risk-adjusted dynamics is

$$
d r_{t}=\kappa\left(\theta-r_{t}\right) d t+\sigma_{r} \sqrt{r_{t}} d W_{t}^{r},
$$

where $r_{0}>0, \kappa, \theta$ and $\sigma_{r}>0$ are constant parameters, and $W^{r}$ is a standard Wiener process. The parameters $\theta$ and $\kappa$ are the long-term mean value of $r$ and the speed of adjustment to $\theta$, respectively (see Cox et al. (1985)).

Let $p(t, T)$ denote the price at time $t$ of a default-free ZCB with maturity date $T$. In CIR model, the value of the bond can be calculated using the formula

$$
p(t, T)=A(\tau) e^{-B(\tau) r_{t}},
$$

where $\tau=T-t$,

$$
\begin{aligned}
& A(\tau)=\left[\frac{2 \gamma e^{(\gamma+\kappa)^{\frac{\tau}{2}}}}{2 \gamma+(\gamma+\kappa)\left(e^{\gamma \tau}-1\right)}\right]^{2 \kappa \theta / \sigma_{r}^{2}}, \\
& B(\tau)=\frac{2\left(e^{\gamma \tau}-1\right)}{2 \gamma+(\gamma+\kappa)\left(e^{\gamma \tau}-1\right)},
\end{aligned}
$$

and

$$
\gamma=\sqrt{\kappa^{2}+2 \sigma_{r}^{2}}
$$

Cathcart and El-Jahel (1998) assume, furthermore, that $W^{X}$ and $W^{r}$ are uncorrelated. As an immediate result, the process that governs default is independent of the interest rate process. The debt issuer enters default at the first instant the process $X$ reaches the barrier $H$. As in Longstaff and Schwartz (1995), bondholders receive $1-w$ default-free ZCBs.

In this setting, denote the value at time $t$ of a defaultable ZCB, with maturity $T$, by $v(X, r ; t, T) . v$ satisfies the partial differential equation

$$
\alpha X \frac{\partial v}{\partial X}+\kappa(\theta-r) \frac{\partial v}{\partial r}+X^{2} \frac{\sigma_{X}^{2}}{2} \frac{\partial^{2} v}{\partial X^{2}}+r \frac{\sigma_{r}^{2}}{2} \frac{\partial^{2} v}{\partial r^{2}}-\frac{\partial v}{\partial \tau}=r v
$$

subjected to some conditions (see Cathcart and El-Jahel (1998)). If default has not occurred, then

$$
v(X, r ; T, T)=1 .
$$

The following boundary condition holds,

$$
v(\infty, r ; t, T)=p(t, T) .
$$

In the event of default, $X_{t}=H$, one has

$$
v(H, r ; t, T)=(1-w) p(t, T) .
$$


Moreover, the boundary conditions for $r$ are

$$
\begin{gathered}
v(X, \infty ; t, T)=0, \\
v(X, r ; t, T)<\infty \quad r \rightarrow 0 .
\end{gathered}
$$

Given the hypothesis of the model, the value of a defaultable ZCB is

$$
v(X, r ; t, T)=p(t, T)-w p(t, T) \mathbb{Q}_{t}\left(\tau^{H} \leq T\right),
$$

where $p(t, T)$ is the value of a default-free $\mathrm{ZCB}$ with the same features, which is calculated using formulas (20)-(23) in the CIR model. $\mathbb{Q}_{t}\left(\tau^{H} \leq T\right)$ is the probability of default under the risk-neutral measure.

This probability can be evaluated analytically (see Moraux (2004)):

$$
\mathbb{Q}_{t}\left(\tau^{H} \leq T\right)=\mathcal{N}\left[-\frac{\ln \frac{X_{t}}{H}+\mu \tau}{\sigma_{X} \sqrt{\tau}}\right]+\left(\frac{X_{t}}{H}\right)^{-2 \mu / \sigma_{X}^{2}} \mathcal{N}\left[-\frac{\ln \frac{X_{t}}{H}-\mu \tau}{\sigma_{X} \sqrt{\tau}}\right],
$$

where $\mu=\alpha-\sigma_{X}^{2} / 2, \tau=T-t$, and $\tau^{H}=\inf \left\{t \geq 0: X_{t} \leq H\right\}$.

In this model, credit spreads

$$
c s=-\frac{\ln [v(X, r ; t, T) / p(t, T)]}{T-t}=-\frac{\ln \left[1-w \mathbb{Q}_{t}\left(\tau^{H} \leq T\right)\right]}{T-t}
$$

do not depend on the level of interest rates.

\subsection{A model with stochastic default boundary and stochastic default-free interest rate}

The model briefly reviewed in this section is a generalization, developed by Saá-Requejo and Santa-Clara (1999) and Hsu et al. (2003), of the model proposed by Nielsen et al. (1993). In these contributions, default-free interest rates are assumed to be stochastic ${ }^{11}$.

An important feature of the model under consideration is the definition of the default boundary $\left(H_{t}\right)$, which is described by a particular diffusion process. $H_{t}$ is the total value at time $t$ of the liabilities (or part of them) in Saá-Requejo and Santa-Clara (1999), while in Hsu et al. (2003) it represents the bankruptcy value of the firm.

In what follows, we consider a firm which not necessarily has a simple capital structure, but may issue a variety of liabilities, also with different maturities. A general assumption that is retained is the simultaneous default on all liabilities.

As in the model proposed by Longstaff and Schwartz (1995), the writedown $w$ is constant and exogenously given ${ }^{12}$. Assume that the recovery value

\footnotetext{
${ }^{11}$ Nielsen et al. (1993) adopt Vasicek model, while in the later works no specific assumption is made about the process that drives the default-free interest rate.

${ }^{12}$ As an alternative, $w(T)$ could be a random variable $\left(\mathcal{F}_{T}\right.$-measurable), not correlated with other processes of the model. When it is assumed constant, $w$ is a parameter that has to be estimated.
} 
upon default is the quantity $1-w$ per units of face value of a default-free ZCB with same maturity.

The value at time $t$ of a ZCB, with maturity at time $T$, can be written as follows ${ }^{13}$

$$
\begin{aligned}
v(t, T) & =\mathbb{E}_{t}\left[\left(1-w \mathbf{1}_{\left\{\tau^{*} \leq T\right\}}\right) e^{-\int_{t}^{T} r(u) d u}\right] \\
& =p(t, T)-\mathbb{E}_{t}\left[w \mathbf{1}_{\left\{\tau^{*} \leq T\right\}} e^{-\int_{t}^{T} r(u) d u}\right],
\end{aligned}
$$

where $p(t, T)$ is the time $t$ value of a default-free $\mathrm{ZCB}, w$ is the writedown, $\tau^{*}$ is the time at which default occurs, and $r$ is the default-free instantaneous interest rate. $\mathbb{E}_{t}(\cdot)$ here means expectation (conditional to the information set at time $t$ ) under a risk neutral measure $\mathbb{Q}$. All the processes in the following discussion are defined with respect to such a risk neutral measure.

Here we consider the simpler model of Saá-Requejo and Santa-Clara (1999) instead that of Hsu et al. (2003).

Let $V$ denote the process describing the assets value of the firm. $V$ evolves according to the stochastic differential equation

$$
d V_{t}=\left(r_{t}-\delta_{V}\right) V_{t} d t+\sigma_{V} V_{t} d W_{t}^{V}, \quad V_{0}>0,
$$

where $\sigma_{V}>0$, and $\delta_{V}$ is the payout rate to the firm's investors. $W^{V}$ is a standard Wiener precess.

The default-free interest rate is assumed stochastic ${ }^{14}$; its uncertainty is driven by a Wiener process $W^{r}$, which is assumed correlated with $W^{V}$, being $\rho_{r V}$ the correlation coefficient.

The default boundary $H=\left(H_{t}\right)_{t \in[0, T]}$ is governed by a stochastic process whose dynamics is described by

$$
\frac{d H_{t}}{H_{t}}=\left(r_{t}-\delta_{H}\right) d t+\sigma_{H r} d W_{t}^{r}+\sigma_{H V} d W_{t}^{V}, \quad H_{0}>0,
$$

where $\sigma_{H r}$ and $\sigma_{H V}$ are two positive constants, $\delta_{H}$ is a payout rate to the firm's debtholders. $H$ is modeled as a joint diffusion process whose uncertainty is related to both $V$ and $r$.

In this setting, default occurs at the first instant the value process $V$ falls below the liabilities value $H$. The default time is then defined as the following first-passage time:

$$
\tau^{*}=\inf \left\{u \geq t: V_{u}=H_{u}\right\}=\inf \left\{u \geq t: V_{u} \leq H_{u}\right\} .
$$

It is convenient to define a new process ${ }^{15}$,

$$
X_{t} \equiv \ln \frac{V_{t}}{H_{t}}=\ln V_{t}-\ln H_{t},
$$

\footnotetext{
${ }^{13}$ At maturity one has $v(T, T)=1-w \mathbf{1}_{\left\{\tau^{*} \leq T\right\}}$.

${ }^{14}$ No particular assumption is made about its dynamics for the moment.

${ }^{15}$ The quantity $V / H$ is called solvency ratio and can be interpreted as a measure of the capability of the firm to repay its debt.
} 
which allows us to write

$$
\tau^{*}=\inf \left\{u \geq t: X_{u}=0\right\} .
$$

The default time is the first instant at which the log-solvency ratio reaches level zero.

We focus on the analysis of the dynamics of the process $X$. It can be shown that $X$, as defined by (37), satisfies the stochastic differential equation ${ }^{16}$

$$
d X_{t}=\mu d t+\sigma d W_{t}^{X} \quad X_{0}>0
$$

with

$$
\begin{gathered}
\mu=\delta_{H}-\delta_{V}-\frac{1}{2}\left(\sigma_{V}^{2}-\left(\sigma_{H V}^{2}+\sigma_{H r}^{2}+2 \rho_{r V} \sigma_{H V} \sigma_{H r}\right)\right), \\
\sigma^{2}=\left(\sigma_{V}-\sigma_{H V}\right)^{2}+\sigma_{H r}^{2}-2 \rho_{r V}\left(\sigma_{V}-\sigma_{H V}\right) \sigma_{H r} .
\end{gathered}
$$

$W^{X}$ is a Wiener process, defined such that

$$
\sigma W_{t}^{X}=\left(\sigma_{V}-\sigma_{H V}\right) W_{t}^{V}-\sigma_{H r} W_{t}^{r},
$$

which is correlated with the Wiener process $W^{r}$, with correlation coefficient

$$
\rho=\frac{\rho_{r V}\left(\sigma_{V}-\sigma_{H V}\right)-\sigma_{H r}}{\sigma} .
$$

In the special case $\rho=0$, default is independent of default-free interest rate; as a result, corporate bonds can be evaluated in closed-form:

$$
v(t, T)=p(t, T)-w p(t, T) \mathbb{Q}_{t}\left(\tau^{*} \leq T\right),
$$

where the probability of default is given by ${ }^{17}$

$$
\mathbb{Q}_{t}\left(\tau^{*} \leq T\right)=\mathcal{N}\left(\frac{-X_{t}-\mu(T-t)}{\sigma \sqrt{T-t}}\right)+e^{-\frac{2 \mu X_{t}}{\sigma^{2}}} \mathcal{N}\left(\frac{-X_{t}+\mu(T-t)}{\sigma \sqrt{T-t}}\right) .
$$

If $\rho \neq 0$, no closed-form solutions are known in general. Saá-Requejo and Santa-Clara (1999) provide approximate solutions in the case of deterministic default-free bond volatilities (as in the Vasicek (1977) model), and suggest to use Monte Carlo simulation in order to calculate an approximation of the default probability in all other cases. As a further result of the simulation, one may compute an estimate of the average time of default.

If we adopt the model of Cox et al. (1985) to modeling the dynamics of $r$, and consider $\rho \neq 0$, we have to jointly generate the paths of the processes

$$
d r_{t}=\left[\kappa\left(\theta-r_{t}\right)+\sigma_{r}^{2} B(\tau) r_{t}\right] d t+\sigma_{r} \sqrt{r_{t}} d W_{t}^{1}
$$

\footnotetext{
${ }^{16}$ See Saá-Requejo and Santa-Clara (1999).

${ }^{17}$ See Bielecki e Rutkowski (2002), Corollary 3.1.1.
} 
and

$$
d X_{t}=\left[\mu+\rho \sigma \sigma_{r} \sqrt{r_{t}} B(\tau)\right] d t+\rho \sigma d W_{t}^{1}+\sqrt{1-\rho^{2}} \sigma d W_{t}^{2},
$$

where $W^{1}$ and $W^{2}$ are two independent Wiener processes. $B(\tau)$ is calculated using formula (22) in the CIR model.

Within this framework, the time $t$ value of the corporate bond is given by

$$
v(t, T)=p(t, T)-w p(t, T) \mathbb{Q}_{t}^{T}\left(\tau^{*} \leq T\right),
$$

where $\mathbb{Q}_{t}^{T}\left(\tau^{*} \leq T\right)$ is the forward risk adjusted probability of default (i.e. the probability measure under which asset values normalized by the price of a default-free ZCB with maturity $T$ are martingales).

This probability can be computed using simulation. To this end, we can use the following numerical scheme:

$$
\begin{aligned}
r_{t_{k+1}} & =r_{t_{k}}\left(1-\kappa \Delta t+\sigma_{r}^{2} B(\tau) \Delta t\right)+\kappa \theta \Delta t+\sigma_{r} \sqrt{r_{t_{k}} \Delta t} z_{k+1}^{1} \quad r_{0}>0, \\
X_{t_{k+1}} & =X_{t_{k}}+\left[\mu+\rho \sigma \sigma_{r} \sqrt{r_{t_{k}+1}} B(\tau)\right] \Delta t+\rho \sigma \sqrt{\Delta t} z_{k+1}^{1}+\sqrt{\left(1-\rho^{2}\right) \Delta t} \sigma z_{k+1}^{2},
\end{aligned}
$$

where $\Delta t=T / n$ is the time step and, for each $k=1, \ldots, n-1$, two independent standard normal variates $\left(z^{1}, z^{2}\right)$ are generated ${ }^{18}$.

\section{Excursion and occupation time models}

First-passage approach does not discriminate between the time at which a firm enters bankruptcy and the time at which it is either liquidated or reorganized: a firm defaults when its assets are too low according to some criterion, and is immediately liquidated. This definition of default no longer reflects economic reality: bankruptcy laws often grant an extended time period for restructuring ${ }^{19}$. In the excursion time approach, discussed in this section, the duration of the default (the time spent in bankruptcy before reorganization or liquidation) is a crucial feature of the model.

Recently there has been some interest in the credit risk literature in models which involve stopping times related to excursions ${ }^{20}$. These models are based on the definitions of Parisian time and occupation time. The Parisian time can be defined as the first instant when a relevant stochastic process (typically a Brownian motion) spends a given amount of time (called the window in the literature related to Parisian options) consecutively beyond

\footnotetext{
${ }^{18} \mathrm{~A}$ simple antithetic variable technique can be used.

${ }^{19}$ Eberhart et al. (1999) investigate the stock return performance of a sample of firms emerging from bankruptcy, and find that the average time spent in bankruptcy (from the default announcement date through the first trading date after distress) is 22.39 months on average. See also Helwege (1999).

${ }^{20}$ See Moraux (2003), Galai et al. (2003), Paseka (2003), Broadie et al. (2004), François and Morellec (2004), Giesecke (2004), Yu et al. (2004), and Nardon (2005).
} 
a certain barrier. This excursion time has been first studied by Chesney et al. (1997) in order to define Parisian barrier options. Parisian options are financial derivatives for which the barrier in- or out-feature is activated when the process driving the underlying price spends continuously a certain amount of time beyond the barrier.

Unlike first-passage time approach, excursion time approach allows for a non-absorbing state of default. Excursion times seem then an attractive tool to define default that occurs when a "grace period" is granted. Moreover, compared to the definition of default time as a first-passage time, this formulation does not suffer from a threshold effect ${ }^{21}$. Even if the relevant process has crossed the barrier, default has not necessarily occurred yet. As a result, the model is more robust than in the "one-touch" case, in which the signaling process need only hit the barrier (no matter how shortly it stays below that threshold) for the default to occur.

Parisian times could then be useful in representing real situations when delays, between the default event and reorganization (or liquidation) of the firm, are involved. This delay could be either a fixed parameter exogenously specified (e.g. prescribed by a debt covenant), or a parameter of the model which has to be estimated from market data. As an alternative, the duration of the default (together with the default boundary) could also be the solution of an optimal decision problem, as in Paseka (2003).

In the following, we assume this delay is constant and let us denote it by $\Delta$. For simplicity, let us consider a constant default boundary, $H$. Suppose also $X_{0}>H$.

Let

$$
\tau_{t}^{H}=\sup \left\{s \leq t: X_{s}=H\right\}
$$

be the last time, before $t$, that the process $X$ hits the threshold $H$.

Define the excursion (Parisian) time

$$
\tau_{\Delta}^{H}=\inf \left\{t \geq 0:\left(t-\tau_{t}^{H}\right) \mathbf{1}_{\left\{X_{t} \leq H\right\}} \geq \Delta\right\} .
$$

The default time is defined as the random time $\tau_{\Delta}^{H}$ and the default probability is

$$
\mathbb{Q}\left[\tau_{\Delta}^{H} \leq T\right]=\mathbb{E}\left[\mathbf{1}_{\left\{\tau_{\Delta}^{H} \leq T\right\}}\right] .
$$

Intuitively, the probability of default in the excursion time approach should fall between the first-passage time case and the default probability in the Black-Scholes-Merton model. When the time window $\Delta$ is zero, we recover the simple first-passage case, while when $\Delta$ is very long and approaches $T$ one expects the probability of default to reduce to that in the Black-Scholes-Merton model. One will check for default only at maturity of the bond.

\footnotetext{
${ }^{21}$ See Haber et al. (1999).
} 
As an example of how excursion times can be introduced in credit risk modeling, consider the model proposed by Çetin et al. (2004). The authors define the process $X$, representing cash balances of the firm, which satisfies the stochastic differential equation

$$
d X_{t}=\sigma d W_{t} \quad X_{0}>0,
$$

with $X_{0}>0, \sigma>0$, and where $W$ is a standard Wiener process. The default threshold is set at zero $(H=0$ in $(51)), \Delta=\frac{\alpha^{2}}{2}$ (for $\alpha>0$ ), so that

$$
\tau_{t}^{H}=\sup \left\{s \leq t: X_{s}=0\right\}
$$

is the last time that the process $X$ hits zero.

$$
\tau_{\Delta}^{H}=\inf \left\{t \geq 0:\left(t-\tau_{t}^{H}\right) \mathbf{1}_{\left\{X_{t}<0\right\}} \geq \Delta\right\},
$$

and the default time is the random time $\tau^{*}$,

$$
\tau^{*}=\inf \left\{t>\tau_{\Delta}^{H}: X_{t}=2 X_{\tau_{\Delta}^{H}}\right\} .
$$

Default occurs, after time $\tau_{\Delta}^{H}$, the first time at which the value of cash balances doubles, after being negative for a long time $(\Delta)$.

Let us turn back to the Parisian time case. Define the barrier time

$$
\Delta_{t}=t-\tau_{t}^{H} \quad t \in[0, T]
$$

as the length of time the process $X$ has spent below the default barrier in the current excursion. $\Delta_{t}$ is simply the difference between the current time $t$ and the last time $X$ hits the barrier. Note that, if the process $X$ is currently above the default threshold, then $\Delta_{t}=0$.

Starting from $X_{0}>H$, the dynamics of $\Delta_{t}$ is described as follows ${ }^{22}$

$$
d \Delta_{t}= \begin{cases}d t & X_{t}<H \\ -\Delta_{t^{-}} & X_{t}=H \\ 0 & X_{t}>H,\end{cases}
$$

where $\Delta_{t^{-}}$is the left limit of $\Delta_{t}$. The "clock" $\Delta_{t}$ is reset to zero when the barrier is reached from below, and it does not change if the process is above the barrier. Default occurs only if the length of the current excursion exceeds a given level, $\Delta_{t} \geq \Delta$, where $\Delta$ is the barrier time parameter. The probability of default is

$$
\mathbb{Q}\left[\tau_{\Delta}^{H} \leq T\right]=\mathbb{Q}\left[\Delta_{t} \geq \Delta\right] .
$$

In the absence of closed-form solutions, the probability $\mathbb{Q}\left[\Delta_{t} \geq \Delta\right]$ can be computed by simulation. The definition of the clock dynamics (59) suggests

\footnotetext{
${ }^{22}$ See Haber et al. (1999).
} 
an easy way to implement the problem. It is well known that the effects of discretization of time can be important when dealing with the evaluation barrier options. The same problems arise when we use Monte Carlo simulation to evaluate the default probability. Nevertheless, this method, even if typically slow and not so accurate, is flexible and easy to implement.

As previously observed, the Parisian time approach does not suffer from a threshold effect, in that a simple hitting of the barrier does not cause default with immediate liquidation of the firm. The triggering of the barrier is fairly robust, nevertheless, this approach suffers from another anomaly. As pointed out by Haber et al. (1999), the resetting of $\Delta_{t}$ is still sensitive to short-term movements of the value process $X$. For example, consider the case of a firm that enters bankruptcy and then, after the process $X$ has spent a long time (but less than $\Delta$ ) under the barrier, it reaches the default threshold (from below) again, before the grace period has elapsed. At the maturity of the bond, the firm will be able to repay its debt, even if it has spent in financial distress almost all the time. As an alternative example, consider a firm that enters and exists bankruptcy more than once during the life of the debt, this without causing liquidation of the firm (note that successive defaults are possible in the economic reality).

To cope with these anomalies, Haber et al. (1999) and Moraux (2003) suggest to consider the cumulative excursion (or occupation) time instead of the continuous excursion time ${ }^{23}$. Moraux (2003) points out that only occupation time models take into account the whole past financial distress of the firm, even if this approach may not be appropriate for very long term bonds. Such an approach considers the total time (over the monitored time interval) spent by the value process beyond the default threshold, hence it seems more suitable in order to study the financial history and distress periods of the debt issuer.

Formally, if $H$ is the barrier, $T$ the maturity of the bond, and $\Delta$ is the maximum duration of time allowed below the barrier, the occupation time is defined as follows

$$
\Delta_{0}^{T}=\int_{0}^{T} \mathbf{1}_{\left\{X_{t} \leq H\right\}} d t
$$

$\Delta_{0}^{T}$ is a measure of the amount of time the process $X$ spends below the barrier during the time interval $[0, T]$. The probability of default is defined by

$$
\mathbb{Q}\left[\Delta_{0}^{T} \geq \Delta\right]=\mathbb{E}\left[\mathbf{1}_{\left\{\Delta_{0}^{T} \geq \Delta\right\}}\right] .
$$

In this formulation, the clock is not reset to zero, and the default time is defined as the first instant the process $X$ has spent totally an amount of time $\Delta$ below the default boundary,

$$
\tau^{*}=\inf \left\{t \geq 0: \Delta_{0}^{t} \geq \Delta\right\} .
$$

\footnotetext{
${ }^{23}$ Options linked to occupation time are also called "ParAsian" options in Haber et al. (1999). See also Hugonnier (1999).
} 
Also in this case, we can use Monte Carlo simulation in order to compute an estimate of the average time of default and the default probability; by exploiting the definition of the clock's dynamics (59), the problem can be implemented in a very intuitive way.

It worths noting that the first-passage approach is more favorable to bondholders, while the excursion time approach, allowing for a grace period, favors firm's rights. Occupation time approach falls, in a certain sense, between the classical first-passage and the Parisian time approaches.

\section{Excursions height and length: an application to credit risk}

Gauthier (2002) studies the stopping time defined by the first instant when a Brownian motion either spends consecutively more than a certain time above a given level, or reaches another level. This stopping time generalizes both the simple hitting time and the Parisian time. Gauthier derives the Laplace transform of this stopping time and applies the obtained results to the valuation of investment projects with a delay constraint and the possibility of starting immediately the project but at a higher cost.

In this section, by exploiting Gauthier's idea, we define the default time as the first instant when a relevant process either stays continuously for a certain time interval under the default boundary, or hits another lower threshold. This could correspond, for instance, to a real situation in which a firm is allowed temporarily to be short of funds, but enters default immediately when the financial distress becomes severe. If either the firm's negative cash flows persist for an extended period of time, or the firm exhausts all its lines of credit and liquid assets, it defaults right away.

More precisely, we are interested in the first time $\tau^{*}$ when the process governing default, $\left(X_{t}\right)_{t \geq 0}$, either spends consecutively a given period of time $\Delta$ (with $\Delta<T$ ) below the default boundary $H$, or reaches the lower threshold $H^{-}<H$.

Let, as in the previous section,

$$
\tau_{t}^{H}=\sup \left\{s \leq t: X_{s}=H\right\}
$$

be the last time, before $t$, that the process $X$ hits the barrier $H$.

Define the excursion time

$$
\tau_{\Delta}^{H}=\inf \left\{t \geq 0: \Delta_{t} \mathbf{1}_{\left\{X_{t} \leq H\right\}} \geq \Delta\right\},
$$

where $\Delta_{t}=t-\tau_{t}^{H}$, and the hitting time

$$
\tau^{H^{-}}=\inf \left\{t \geq 0: X_{t} \leq H^{-}\right\} .
$$


Figure 1: Instances of default time in the approach based on excursions height and length.

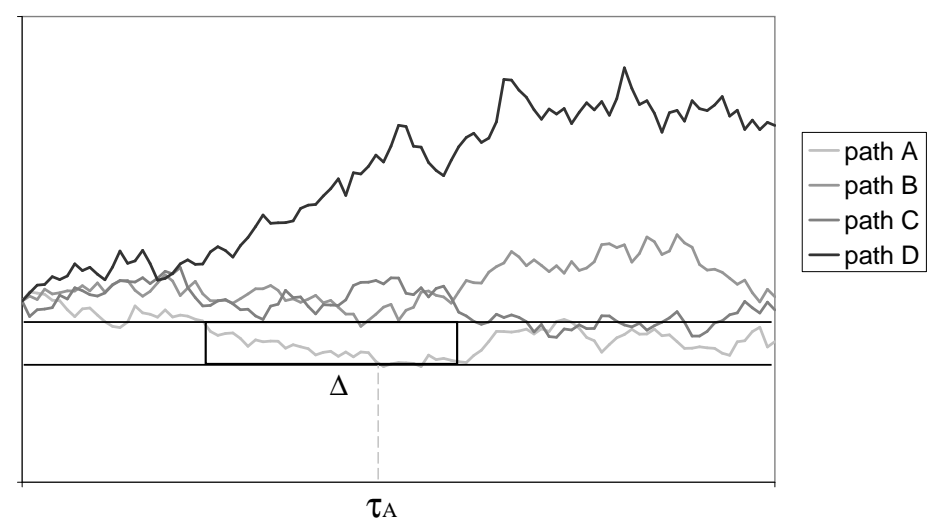

In this formulation, the default time is

$$
\tau^{*}=\min \left(\tau_{\Delta}^{H}, \tau^{H^{-}}\right),
$$

and the probability of default is

$$
\mathbb{Q}\left[\tau^{*} \leq T\right]=\mathbb{E}\left[\mathbf{1}_{\left\{\tau^{*} \leq T\right\}}\right] .
$$

The definition of the stopping time $\tau^{*}$ in (67) extends both the excursion time and the first-passage time definitions. $\tau^{*}$ is the first time the process $X$ leaves the region defined by the default boundary $H$ on top and the lower boundary $H^{-}$on bottom, and length $\Delta$, without reaching the barrier $H$ again from below. Credit risk models based on excursions height and length account for both the severity (height) and persistency (length) of the financial distress (the excursion).

Let us consider, for simplicity, constant boundaries. As an alternative, we could also model at $H$ and $H^{-}$as time-varying barriers, like in the Black and Cox (1976) model. In this case, we could define

$$
H_{t}=H e^{-\gamma(T-t)},
$$

and

$$
H_{t}^{-}=H e^{-\eta(T-t)},
$$

with parameters $\gamma<\eta$, and $H>0$ (typically set equal to the face value of the debt $F$ ). In order to avoid inconsistencies, the event of default at maturity has to be defined carefully, as discussed in Section 2 . 
Figure 1 shows an example of excursion in the distress region: default occurs only for path $A$ at time $\tau_{A}$.

These boundaries could also be endogenously determined, like in the model proposed by Paseka (2003) (which is similar to the approach here presented), in which default occurs at the time

$$
\tau^{B}=\inf \left\{t>0: X_{t}=H^{B}\right\}
$$

where $H^{B}>0$ is the bankruptcy barrier (the level at which the firm enters bankruptcy). Liquidation occurs only if the reorganization is unsuccessful, and this happens at time

$$
\tau^{L}=\inf \left\{t>0: X_{t}=H^{L}\right\},
$$

where $H^{L}<H^{B}$ is the liquidation barrier. Otherwise, if the value of the firm rises to a certain level $H^{R}$ (which represents the reorganization boundary) at which a reorganization plan is proposed by the debtor, the firm exits default. Define the random time

$$
\tau^{R}=\inf \left\{t>0: X_{t}=H^{R}\right\}
$$

then the timing of exit from bankruptcy is the minimum between the reorganization time and the liquidation time

$$
\tau^{*}=\tau^{R} \wedge \tau^{L} .
$$

In Paseka's model, the level $H^{L}$ is exogenously specified, while the boundaries $H^{B}$ and $H^{R}$ are both determined endogenously in order to maximize equity value. As a result, also the duration of bankruptcy is a solution of a bargaining process.

The idea of modeling excursions in space and time for valuing corporate debt could be generalized by introducing multiple boundaries at which the firm value process must take on specific values. In such a framework, the triggering of a barrier signals some credit event like, for example, a credit migration (hence a downgrade or an upgrade in rating of the issuer). To this regard, Esteghamat (2003) points out that "boundaries in the space separate credit events", and proposes a model which connects structural and credit rating models by treating credit events as crossings of a multidimensional stochastic process through bounded regions in a probability space.

A further generalization of the approach based on excursions height and length, and related stopping times, is the idea of weighting large deviations below the default barrier more strongly, in order to take into account the severity of the financial distress ${ }^{24}$. As suggested by Haber et al. (1999), in

\footnotetext{
${ }^{24}$ Models based on a weighted definition of the distress clock have been proposed by Galai et al. (2005), Giesecke (2004), and Nardon (2005).
} 
the modeling of financial options with Parisian features, the speed at which the clock process $\Delta_{t}$ changes could be proportional to the distance of the value process $X$ from the default barrier. Another possible specification consists in considering upper boundaries together with lower boundaries: the time spent above the default threshold might be subtracted from the time spent below it. This latter formulation could be useful in modeling situations in which a firm alternates periods of fund shortages to excess of liquidity. One can easily envisage many other possible stylized situations.

\section{Analysis of some numerical examples}

In this section we examine the effects of different default time specifications on bond prices and credit spreads, under the assumptions of one of the structural models proposed in the literature. In particular, in the simulation analysis, we adopt the model proposed by Cathcart and El-Jahel (1998) briefly reviewed in Section 2.

In order to estimate the default probability we used analytical formulas (when available) and Monte Carlo simulation in all other cases. As already observed, the idea of modeling the time of default as a hitting or excursion time is applied in a very easy and intuitive manner in the simulation. One generates a sufficiently high number of trajectories for the process $X$ that signals default, and the default probability is simply approximated by the relative frequency of the default triggering event.

In the experiments carried out, we generated 100000 paths (50000 antithetic) of the process $X$. We considered a time step equal to $\Delta t=1 / 250$ (which approximatively corresponds to daily observations of the process $X$ ) and bond maturities (in years) in the range $[0,20]$.

Figure 2 shows the bond prices (where $p$, represented by a dotted line, is the value of the default-free bond) and the term structure of credit spreads for different values of the writedown $w$ and for different specifications of the default time. More precisely, in the first case shown in Figure 2, we used the original model of Cathcart and El-Jahel (1998). In the second case reported, we introduce a delay of $\Delta=0.5$ (six months), while in the third case we consider the cumulative excursion time. In the fourth case, we introduce both the grace period $\Delta$ and a lower boundary $H^{-}=0.9 H$.

The term structure of credit spreads displays the typical humped shape. We observe that, the introduction of a delay in the timing of default, has the effect of changing the shape and level of credit spreads. When the default barrier is assumed constant, as in this case, and being other model factors unchanged, we obtain higher bond values and then lower credit spreads. This result is also intuitive. Observing the graphics in Figure 2, it seems that different hypothesis made on the excursions have no such an effect on the spreads. This is, actually, due to fact that the process $X$ has continuous 
paths and "takes some time" to reach the lower barrier $H^{-}$(which is set at $90 \%$ of the value of $H$ ). On the other hand, what changes is the average time of default, and the probability that default occurs before or at maturity.

Figure 3 shows another similar experiment, in which we consider a higher ratio $X_{0} / H=2$. We report only the results for the term structure of credit spreads. While in Figure 4 we let the ratio $X_{0} / H$ vary and held other parameters constant.

\section{Conclusions}

A variety of approaches have been proposed in the literature with the aim of evaluating defaultable debt and trying to better explain the process that drives default. In this contribution we first examined first-passage time models of defaultable bond pricing in which default occurs at the first time a relevant process reaches the so-called default boundary. First-passage approach makes no distinction between default and liquidation. Recently there has been increasing interest in the credit risk literature in models which involve stopping times related to excursions. This relatively new approach has been discussed, pointing out its advantages and disadvantages.

We then proposed a generalization of both the hitting time and the excursion time approaches, which consists in defining the default time as the first instant at which the firm value process (or another signaling process) either has spent a certain time below the default threshold or hits a lower barrier. In the present work, we focused more on the different definitions of the timing of default, rather than on other important and interesting issues (which require further research) like the specification of the default boundary, the dynamics of the process that governs default, the recovery value upon default, the dynamics of the default-free interest rate and its correlation with the process that signals default. On the other hand, the idea of modeling the timing of default as either a hitting or excursion time is potentially applicable to a very large class of models.

Finally, we then examined the effects of different default time specifications on bond prices and credit spreads within a stylized model. As a result of the numerical analysis carried out, we observed that the introduction of a delay in the timing of default has an impact on credit spreads. When the default barrier is assumed constant, and being other model factors unchanged, we obtain (as expected) higher bond values and, hence, lower credit spreads.

Observing the term structure of credit spreads obtained in the numerical experiments, one can notice that at short maturities the issuer has no or very low default risk, after which, conditional on no earlier default, credit spreads rises (also significantly in some cases). Many authors point out that such term structures of default probabilities and credit spreads are not realistic. The excursion approach retains (and even stresses) this characteristic which 
is common to first-passage time models, based on a diffusion process that drives default, and perfect knowledge of the firm's value and default boundary. Even thought an issuer may be of poor credit quality the probability of default within a short time horizon is very low. Models of firm dynamics that allow for jumps, or imperfect information (of course at the expense of some tractability), would generate term structure of credit spreads and default probabilities more in accord with observed data. This important issue is left for future research.

\section{References}

[1] Bielecki, T. R. and M. Rutkowski, 2002. Credit Risk: Modeling, Valuation and Hedging. (Springer-Verlag, Berlin).

[2] Black, F. and J. C. Cox, 1976. Valuing corporate securities: some effects of bond indeture provisions. Journal of Finance, 31, 351-367.

[3] Briys, E. and F. de Varenne, 1997. Valuing fixed rate debt: An extention. Journal of Financial and Quantitative Analysis, 32, 239-248.

[4] Broadie, M., M. Chernov and S. Sundaresan, 2004. Optimal debt and equity values in the presence of Chapter 7 and Chapter 11. Working Paper.

[5] Cathcart, L. and L. El-Jahel, 1998. Valuation of defaultable bonds. Journal of Fixed Income, 8 (1), 65-78.

[6] Çetin, U., R. Jarrow, P. Protter, and Y. Yildirim, 2004. Modeling credit risk with partial information. Annals of Applied Probability, 14 (3), $1167-1178$.

[7] Chesney, M., M. Jeanblanc-Picqué and M. Yor, 1997. Brownian excursions and Parisian barrier options. Advances in Applied Probability, 29, $165-184$.

[8] Collin-Dufresne, P. and R. S. Goldstein, 2001. Do credit spreads reflect stationary leverage ratios? Journal of Finance, 56, 1929-1958.

[9] Cox, J. C., J. E. Ingersoll and S. Ross, 1985. A theory of the term structure of interest rates. Econometrica, 53, 385-407.

[10] Duffie, D. and D. Lando, 2001. Term structures of credit spreads with incomplete accounting information. Econometrica, 69, 633-664.

[11] Eberhart, A. C., E. I. Altman and R. Aggarwal, 1999. The equity performance of firms emerging from bankruptcy. Journal of Finance, 54, $1855-1868$. 
[12] Esteghamat, K., 2003. A boundary crossing model of counterparty risk. Journal of Economic Dynamics \& Control, 27, 1771-1799.

[13] François, P. and E. Morellec, 2004. Capital structure and asset prices: some effetcs of bankruptcy procedures. Journal of Business, 77 (2), $387-411$.

[14] Galai, D., A. Raviv and Z. Wiener, 2003. Liquidation triggers and the valuation of equity and debt. Working Paper.

[15] Gauthier, L., 2002. Excursions height- and length-related stopping times, and application to finance. Advances in Applied Probability, 34, 846-868.

[16] Giesecke, K., 2004. Credit risk modeling and valuation: An introduction. Working Paper, Cornell University.

[17] Guha, R. and A. Sbuelz, 2003. Structural RFV: Recovery form and defaultable debt analysis. Working Paper.

[18] Haber, R. J., P. Schönbucher and P. Wilmott, 1999. Pricing Parisian options. Journal of Derivatives, 6 (3), 71-79.

[19] Helwege, J., 1999. How long do junk bonds spend in default? Journal of Finance, 54, 341-357.

[20] Hsu, J. C., J. Saá-Requejo and P. Santa-Clara, 2003. Bond pricing with default risk. Working Paper.

[21] Hugonnier, J., 1999. The Feynman-Kac formula and pricing occupation time derivatives. International Journal of Theoretical and Applied Finance, 2 (2), 153-178.

[22] Hui, C. H., C. F. Lo and S. W. Tsang, 2003. Pricing corporate bond with dynamic default barriers. Journal of Risk, 5 (3), 17-39.

[23] Jarrow, R. A. and P. Protter, 2004. Structural versus reduced form models: a new information based perspective. Journal of Investment Management, 2 (2), 1-10.

[24] Kim, I. J., K. Ramaswamy and S. Sundaresan, 1993. Does default risk coupons affect the valuation of corporate bonds? A contingent claims model. Financial Management, 22 (3), 117-131.

[25] Leland, H. E., 1994. Corporate debt value, bond covenants, and optimal capital structure. Journal of Finance, 59, 1213-1252.

[26] Leland, H. E. and K. Toft, 1996. Optimal capital structure, endogenous bankruptcy, and the term structure of credit spreads. Journal of Finance, 51, 987-1019. 
[27] Longstaff, F. A. and E. S. Schwartz, 1995. A simple approach to valuing risky fixed and floating rate debt. Journal of Fianance, 50, 789-819.

[28] Merton, R. C., 1974. On the pricing of corporate debt: the risk structure of interest rates. Journal of Finance, 29, 449-470.

[29] Moraux, F., 2003. Valuing corporate liabilities when the default threshold is not an absorbing barrier. Working Paper, Université de Rennes I - IGR.

[30] Moraux, F., 2004. A close form solution for pricing defaultable bonds. Finance Research Letters, 1, 135-142.

[31] Nardon, M., 2005. Valuing defaultable bonds: an excursion approach. Rendiconti per gli Studi Economici Quantitativi, Issue 2005, 195-210.

[32] Nielsen, L. T., J. Saá-Requejo and P. Santa-Clara, 1993. Default risk and interest rate risk: the term structure of default spreads. Working Paper, INSEAD.

[33] Paseka, A., 2003. Debt valuation with endogenous default and Chapter 11 reorganization. Working Paper.

[34] Protter, P., 2004. Stochastic Integration and Differential Equations. (Springer-Verlag, New York).

[35] Saá-Requejo, J. and P. Santa-Clara, 1999. Bond pricing with default risk. Working Paper.

[36] Schmid, B., 2004. Credit Risk Pricing Models: Theory and Practice. (Springer-Verlag, Berlin).

[37] Shimko, D., N. Tejima and D. R. van Deventer, 1993. The pricing of risky debt when interest rates are stochastic. Journal of Fixed Income, $3(2), 58-65$.

[38] Taurén, M. P., 1999. A model of corporate bond prices with dynamic capital structure. Working Paper.

[39] Vasicek, O., 1977. An equilibrium characterization of the term structure. Journal of Financial Economics, 5, 177-188.

[40] Yu, L. Z., D. P. Newton, P. W. Duck, M. Widdicks and P. V. Johnson, 2004. Pricing credit risk as ParAsian options with endogenous recovery rate of corporate bonds. Working Paper. 
Figure 2: Defaultable ZCB and default-free ZCB (dotted line) prices and the term structure of credit spreads for different values of the writedown $w \in$ $\{0.25,0.5,0.75,1\}$, in the first-passage, excursion (with $\Delta=0.5$ ), cumulative excursion, and excursion in height and length (with $H^{-}=0.9 H$ ) cases. The other parameters are: $\alpha=0.02, \sigma_{X}=0.2, X_{0} / H=1.5, r_{0}=0.02, \theta=0.04$, $\kappa=0.5, \sigma_{r}=0.03$.
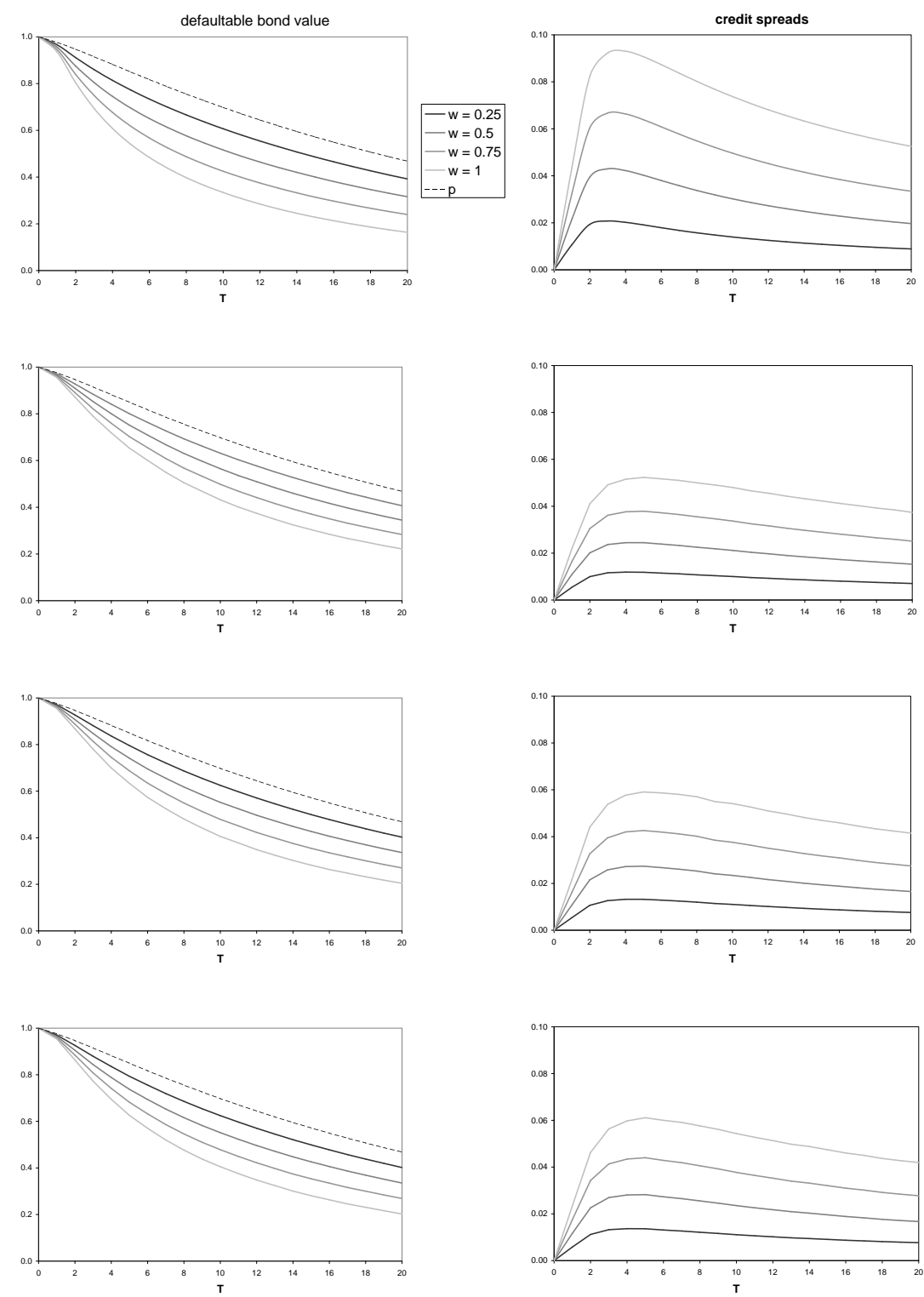
Figure 3: The term structure of credit spreads for different values of the writedown $w \in\{0.25,0.5,0.75,1\}$, in the first-passage, excursion (with $\Delta=$ 0.5 ), cumulative excursion, and excursion in height and length (with $\mathrm{H}^{-}=$ $0.9 \mathrm{H}$ ) cases. The other parameters are: $\alpha=0.02, \sigma_{X}=0.2, X_{0} / H=2$, $r_{0}=0.02, \theta=0.04, \kappa=0.5, \sigma_{r}=0.03$.
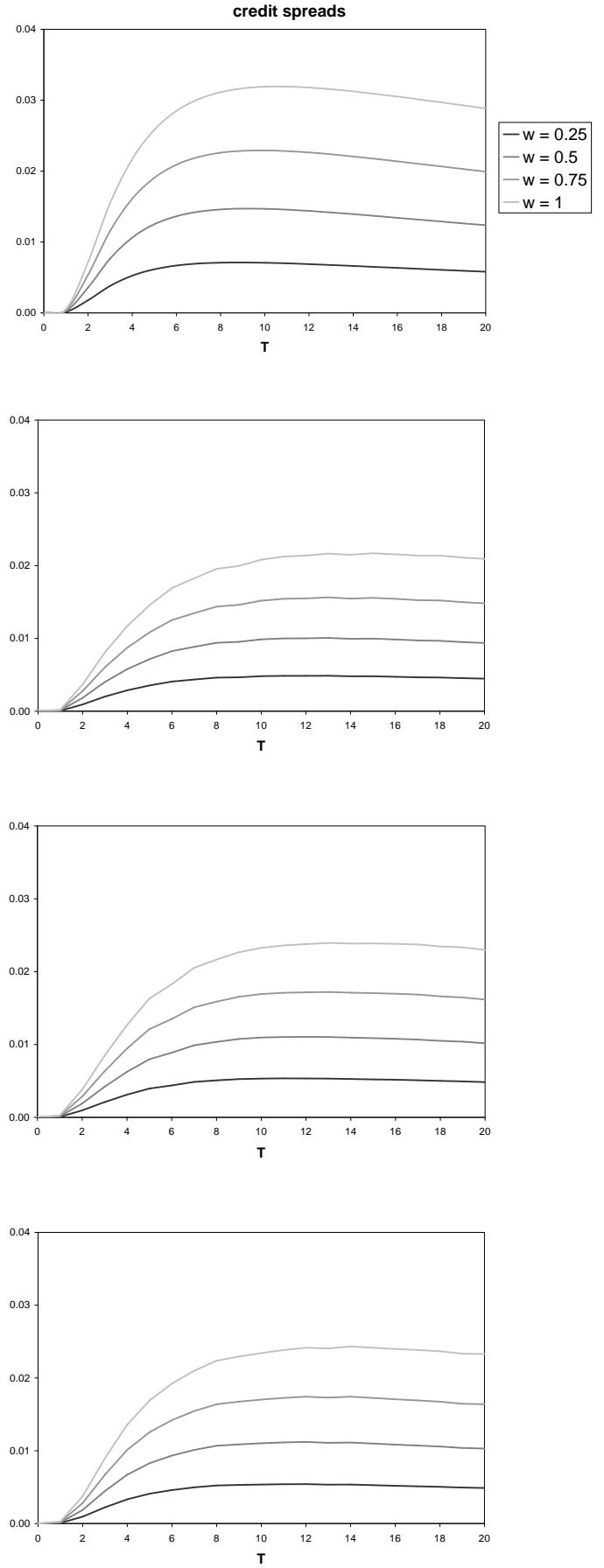
Figure 4: The term structure of credit spreads for different values of the ratio $X_{0} / H \in\{1.5,2,2.5\}$, in the first-passage, excursion (with $\Delta=0.5$ ), cumulative excursion, and excursion in height and length (with $\mathrm{H}^{-}=0.9 \mathrm{H}$ ) cases. The other parameters are: $\alpha=0.02, \sigma_{X}=0.2, w=0.5, r_{0}=0.02$, $\theta=0.04, \kappa=0.5, \sigma_{r}=0.03$.
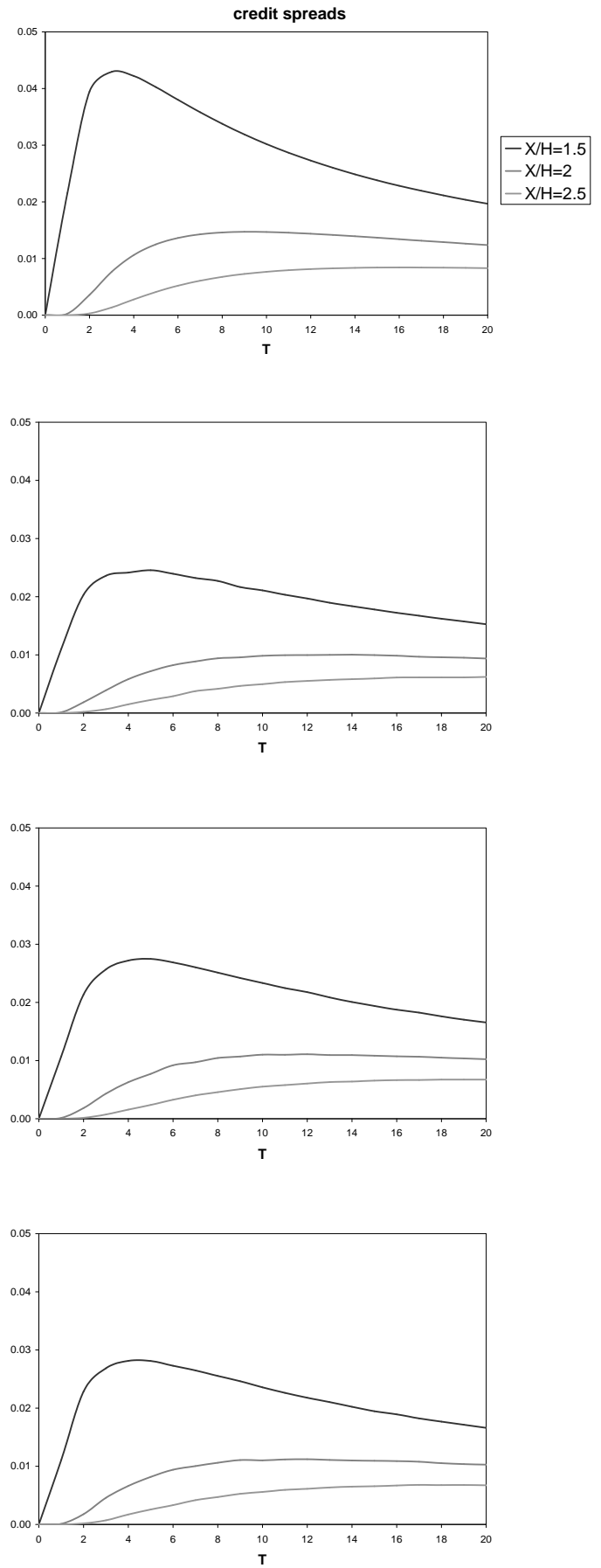